J Mater Chem B Mater Biol Med. 2017 July 7; 5(25): 5019-5026. doi:10.1039/C7TB00598A.

\title{
POSS-ProDOT Crosslinking of PEDOT
}

\author{
Bin Wei ${ }^{\mathrm{a}}$, Jinglin Liu ${ }^{\mathrm{a}}$, Liangqi Ouyang ${ }^{\mathrm{a}}$, and David C. Martin ${ }^{\mathrm{a}, \mathrm{b}}$ \\ aMaterials Science and Engineering, the University of Delaware, Newark, DE, USA,19716 \\ bBiomedical Engineering, the University of Delaware, Newark, DE, USA, 19716
}

\section{Abstract}

\begin{abstract}
Alkoxy-functionalized polythiophenes such as poly(3,4-ethylenedioxythiophene) (PEDOT) and poly(3,4-propylenedioxythiophene) (PProDOT) have become promising materials for a variety of applications including bioelectronic devices due to their high conductivity, relatively soft mechanical response, good chemical stability and excellent biocompatibility. However the longterm applications of PEDOT and PProDOT coatings are still limited by their relatively poor electrochemical stability on various inorganic substrates. Here, we report the synthesis of an octaProDOT-functionalized polyhedral oligomeric silsesquioxane (POSS) derivative (POSS-ProDOT) and its copolymerization with EDOT to improve the stability of PEDOT coatings. The POSSProDOT crosslinker was synthesized via thiol-ene "click" chemistry, and its structure was confirmed by both Nuclear Magnetic Resonance and Fourier Transform Infrared spectroscopies. PEDOT copolymer films were then electrochemically deposited with various concentrations of the crosslinker. The resulting PEDOT-co-POSS-ProDOT copolymer films were characterized by Cyclic Voltammetry, Electrochemical Impedance Spectroscopy, Ultraviolet-Visible spectroscopy and Scanning Electron Microscopy. The optical, morphological and electrochemical properties of the copolymer films could be systematically tuned with the incorporation of POSS-ProDOT. Significantly enhanced electrochemical stability of the copolymers was observed at intermediate levels of POSS-ProDOT content (3.1 wt\%). It is expected that these highly stable PEDOT-coPOSS-ProDOT materials will be excellent candidates for use in bioelectronics devices such as neural electrodes.
\end{abstract}

\section{Introduction}

Conjugated polymers have gained much interest in a wide range of bioelectronic applications, such as biosensors, drug delivery, tissue engineering, actuators, and neural interfaces. ${ }^{1-8}$ These polymers are particularly attractive for neural interface applications due to their relatively soft mechanical properties, tunable surface morphology, high conductivity and excellent biocompatibility. ${ }^{9-11}$ These soft materials usually have a typical Young's modulus on the order of $\sim 1 \mathrm{GPa}$ and thus are expected to help reduce the mechanical properties mismatch between hard metal electrodes and soft living tissue. ${ }^{12,13}$ In addition,

Correspondence to: David C. Martin.

$\dagger$ Electronic Supplementary Information (ESI) available: See DOI: 10.1039/x0xx00000x

Notes: D.C.M is a Co-Founder and Chief Scientific Officer for Biotectix LLC, a University of Michigan spin-off company working to develop conjugated polymers for interfacing a variety of electronic biomedical devices with living tissue. 
they provide high surface areas that can facilitate ion exchange between the microelectrodes and tissue, therefore building more efficient pathways for abiotic-biotic communication. ${ }^{14}$ Because of their good electronic and ionic conductivities, the impedance is usually significantly reduced and charge storage capacity is substantially improved. Among the currently available conjugated polymers, alkoxy-functionalized polythiophenes such as poly(3,4-ethylenedioxythiophene) (PEDOT) and poly(3,4-propylenedioxythiophene) (PProDOT) have become particularly promising candidates because of their low oxidation potential, relatively high chemical and thermal stability, and high conductivity. ${ }^{15,16}$ PEDOTcoated electrodes have been widely studied for use in neural interfaces, and their advantages and improvements over conventional metal electrodes have been reported. ${ }^{17-19}$ Though conjugated polymers have many advantages over other materials, these materials are often fragile and may delaminate from the surfaces of metal electrodes. ${ }^{12}$ Another concern is their electrochemical and mechanical stability for long-term neural recording or stimulation applications. Loss of device performance such as decreased charge carrying capacity and increased impedance of PEDOT coated neural electrodes under chronic neural recording or stimulation conditions has been reported by different groups. ${ }^{20,21}$ Not only do these delaminations potentially decrease the charge transfer performance of the devices, but they could also lead to residual fractions of the film left behind in the tissue. It is therefore of interest to make more electrochemically and mechanically stable PEDOT films to improve their long-term performance and minimize the uncertainties this may pose to biological systems.

Several strategies have been directed toward improving the electrochemical stability of conjugated polymers on metallic substrates. Cui et al. found that stability of PEDOT films was improved by using carbon nanotubes as dopants during electrochemical polymerization. ${ }^{3}$ Inganas and coworkers chemically crosslinked the polyelectrolyte dopant PSS and found that the stability of PEDOT: PSS was substantially improved. ${ }^{22,23}$

Khodagholy et al. have shown that glycidoxypropyltrimethoxysilane (GOPS) can be used to improve the adhesion and long-term stability of spuncast PEDOT:PSS, while retaining effective charge transport performance. ${ }^{24}$ The ability to make more mechanically stable PEDOT films by using adhesion promoters has been reported by Wei et al. ${ }^{25}$ and Carli et al. $^{26}$

Despite these developments, the search for conducting polymer materials that are more electrochemically and mechanically robust remains an ongoing topic of interest. Recently Ouyang et al. reported the use of a conjugated 3-armed EDOT derivative, 1,3,5-tri[2-(3,4ethylenedioxythienyl)]-benzene ( $\mathrm{EPh})$ as a crosslinker to increase the stability of PEDOT coatings. It was found that the stiffness and fracture resistance were both improved by adding EPh to PEDOT. However the EPh monomer disrupted the conjugation of the chain backbone, leading to dramatic changes in color and a corresponding decrease of the charge transport efficiency. It would therefore be useful to find crosslinking agents for PEDOT that could increase the mechanical performance yet not lead to such a dramatic drop in charge transport properties.

Here, we introduce the use of a ProDOT functionalized polyhedral oligomeric silsesquioxane (POSS) that substantially increases the stability of PEDOT films, while 
retaining its ability to efficiently transport charge. We took advantage of the cubic POSS cage moiety that consists of 8 silicon atoms at the corners with oxygens along the cube edges (Scheme 1). The POSS core can be chemically functionalized with various organic substituents attached to the silicon corner atoms, forming an organic-inorganic hybrid framework when incorporated into other polymer matrices. ${ }^{27-29}$ Functionalized POSS nanomaterials have been previously shown to enhance temperature and oxidation resistance, surface hardening and mechanical integrity of a variety of polymers. For example, the incorporation of POSS units was shown to significantly enhance the electrochemical performance of polyaniline, as shown by cyclic stability testing. ${ }^{27}$

In this work, we synthesized a functionalized POSS-ProDOT crosslinker via hydrosilylation and thiol-ene "click" chemistry, and subsequently prepared PEDOT-co-POSS-ProDOT copolymers of various compositions by electrochemical polymerization. Since the multifunctional POSS-ProDOT used in this study keeps the reactive thiophene units well away from the center of the crosslinking agent, we anticipated that it would be able to provide the desired increase in electrochemical and mechanical performance without necessarily causing significant disruption in the conjugation of the chains themselves. We expected that the POSS-ProDOT crosslinker would enhance the long-term electrochemical stability of PEDOT coatings while preserving the excellent electrical properties of PEDOT. Our results show that small amounts of POSS-ProDOT (3.1 wt $\%$ ) do indeed provide substantial improvements in stability without sacrificing toughness or electrical transport performance. However further additions cause the films to become more brittle.

\section{Experimental}

\subsection{Materials}

3,4-dimethoxythiophene, diethyl allylmalonate, lithium aluminum hydride, p-toluene sulfonic acid (p-TSA), tetrabutyl ammoniumperchlorate (TBAP), 3-Mercaptopropyl trimethoxysilane (MTS),2,2-dimethoxy-2-phenylacetophenone (DMPA) and 3,4ethylenedioxythiophene (EDOT) were purchased from Sigma-Aldrich. 2-allyl-1,3propanediol was synthesized from dietyhyl allylmalonate. ${ }^{15}$ Stainless steel electrodes (E363/76/SPC) were purchased from Plastics One. ITO coated glass slides were purchased from Delta Technologies with a surface resistivity of $4-8 \Omega / \mathrm{sq}$. All other chemicals were of analytical grade, and Milli-Q water from a Millipore $\mathrm{Q}$ water purification system was used throughout. All reagents and solvents were used without further purification, unless otherwise noted. Samples were irradiated using a UVP Black Ray UV Bench Lamp $\mathrm{XX}-15 \mathrm{~L}$, emitting $365 \mathrm{~nm}$ light at $15 \mathrm{~W}$.

\subsection{Syntheses of alkene functionalized ProDOT derivative (ProDOT-ene) and POSS-SH}

ProDOT-ene was synthesized via the p-TSA catalyzed transetherification route from 3,4dimethioxythiophene and 2-allyl-1,3-propanediol (Scheme $1 \mathrm{~b}$ ) as previously described. ${ }^{15}$ 1H NMR (400 MHZ, $\left.\mathrm{CDCl}_{3}\right): \delta=6.48(\mathrm{~s}, 2 \mathrm{H}) ; 5.78(\mathrm{~m}, 2 \mathrm{H}) ; 5.10(\mathrm{~m}, 1 \mathrm{H}) ; 4.10-4.16(\mathrm{~m}$, $2 \mathrm{H}) ; 3.86-3.92(\mathrm{~m}, 2 \mathrm{H}) ; 2.18-2.26(\mathrm{~m}, 3 \mathrm{H})$. 
The synthesis of POSS-SH is shown in Scheme 1a according to literature. $5 \mathrm{~mL}$ MTS, 10 $\mathrm{mL}$ concentrated hydrochloric acid and $120 \mathrm{~mL}$ methanol were added into a $500 \mathrm{~mL}$ round bottom flask. The reaction mixture was refluxed at $90^{\circ} \mathrm{C}$ for $30 \mathrm{~h}$ under agitation to ensure the completed hydrosilylation. The white viscous precipitate was first washed with methanol for three times and then dissolved in $2 \mathrm{~mL}$ THF. The THF solution was added to $100 \mathrm{~mL}$ acetonitrile drop wisely and then allowed to crystallize at $-20{ }^{\circ} \mathrm{C}$ for overnight. The final product was dried in a vacuum oven at room temperature for $12 \mathrm{~h}$, and the yield was $59 \% .{ }^{1} \mathrm{H}$ NMR (600 MHZ, $\left.\mathrm{CDCl}_{3}\right): \delta=2.56(\mathrm{~s}, 2 \mathrm{H}) ; 1.72(\mathrm{~s}, 2 \mathrm{H}) ; 1.38(\mathrm{~s}, 1 \mathrm{H}) ; 0.77(\mathrm{~m}$, $2 \mathrm{H})$.

\subsection{Preparation of POSS-ProDOT via thiol-ene "click" chemistry}

POSS-ProDOT was synthesized from POSS-SH and ProDOT-ene. POSS-SH (30 mg, 0.03 $\mathrm{mmol}$ ) and a minimum of THF were added to a vial and heated to dissolve the thiol. ProDOT-ene (70 $\mathrm{mg}, 0.35 \mathrm{mmol}$ ) and $0.1 \mathrm{wt} \%$ of 2,2-dimethoxy-2-phenylacetophenone (DMPA) were added, and the mixture was sparged with argon for five minutes. The vial was placed under a UV lamp and irradiated for one hour (Scheme 1c). A small amount of methanol was added, and the product was ultrasonicated for $2 \mathrm{~min}$. The supernatant was decanted and the product redissolved in chloroform, and concentrated by evaporation to give a viscous oil. The yield of the thiol-ene reaction was $66 \%$. $1 \mathrm{H} \mathrm{NMR} \mathrm{(600} \mathrm{MHZ,} \mathrm{CDCl3):} \delta=$ 6.48 (s, 2H); 4.11 (m, 2H); 3.89 (m, 2H); 2.52 (s, 4H); $2.14(\mathrm{~m}, 1 \mathrm{H}) ; 1.67$ (s, 4H); 1.54 (m, $2 \mathrm{H}) ; 0.77$ (s, 2H).

\subsection{Electrochemical polymerization and characterization}

All the electrochemical polymerization and characterization experiments were performed with a Gamry Potentiostatic Reference 600TM in a three electrode cell. Stainless steel electrode (Plastics One) or ITO coated glass (Delta Technologies) served as the working electrode, a platinum plate of $1 \mathrm{~cm}^{2}$ surface area was the counter electrode, and $\mathrm{Ag} / \mathrm{AgNO}_{3}$ was the reference electrode. The electrochemical characterization of POSS-ProDOT was studied in a dilute $(2 \mathrm{mM})$ dichloromethane solution containing $0.1 \mathrm{M}$ tetrabutyl ammonium perchlorate (TBAP) as supporting electrolyte. PEDOT homopolymer and PEDOT: POSSProDOT copolymers with various contents of POSS-ProDOT were prepared under constant voltage $(+1.1 \mathrm{~V})$ with a total electrodeposition charge of $27 \mathrm{mC}$. For CV, the polymer films were scanned between $-1.0 \mathrm{~V}$ and $+0.8 \mathrm{~V}$ in a phosphate buffered saline (PBS) buffer solution free of monomer with an $\mathrm{Ag} / \mathrm{AgCl}$ reference electrode. For EIS, the sample acted as the working electrode, a platinum plate as the counter electrode and PBS as the electrolyte. A sinusoidal AC signal with $10 \mathrm{mV}$ amplitude was applied over a frequency range of 1-10 Hz. Each impedance spectrum was obtained at least three times, and the error bars show standard deviations.

\subsection{Characterization}

Nuclear Magnetic Resonance (NMR) spectra $\left({ }^{1} \mathrm{H},{ }^{13} \mathrm{C}\right.$ and $\left.{ }^{29} \mathrm{Si}\right)$ were acquired on either a Bruker DRX-400 or Bruker DRX-600 spectrometer. Chemical shifts are reported in parts per million, referenced to chloroform solvent as internal standard $\left(\mathrm{CDCl}_{3}: 7.24 \mathrm{ppm}\right.$ for ${ }^{1} \mathrm{H}$ and 77.2 for ${ }^{13} \mathrm{C}$ ). Fourier-Transform Infrared (FTIR) spectra were collected on a Perkin Elmer Spectrum 100 spectrometer fitted with a Universal ATR accessory. UV-visible (UV-vis) 
spectra of the polymer films were collected on a Shimadzu UV-2550 spectrophotometer (Shimadzu, Japan), the polymer films were reduced at $-1.0 \mathrm{~V}$ for 1 minute before measurement. Scanning Electron Microscopy (SEM) images of the polymer films were acquired using a Zeiss Auriga 60 Focused Ion Beam Scanning Electron Microscope (FIBSEM) operating at $3 \mathrm{kV}$.

\section{Results and discussion}

\subsection{Syntheses and characterization of POSS-SH, ProDOT-ene and POSS-ProDOT}

Thiol-functionalized polyhedral oligomeric silsesquioxane (POSS-SH)was synthesized via the direct hydrosilylation of 3-mercaptopropyltrimethoxysilane (MTS) in methanol as shown in Scheme 1a. ${ }^{30,31}$ The chemical structure was confirmed by both NMR and FTIR spectra. The thiol functional side groups are critical for the subsequent thiolene "click" reaction to make a core material that can act as crosslinker during the electrodeposition, thus the existence of the thiolgroups needed to be verified first. The ${ }^{1} \mathrm{H}$ NMR spectrum of POSS-SH with assigned peaks is shown in Figure 1a. The thiol side group was clearly confirmed by the triplet centered at $1.38 \mathrm{ppm}$. The presence of the thiol group was also confirmed by an absorption peak locatedat $2550 \mathrm{~cm}^{-1}$ corresponding to the characteristic absorption of the SH stretching vibration in FTIR (Fig. S1). The 3D structure of the POSS-based hybrid material was also confirmed by both NMR and FTIR spectra. The sharp peak located at $-67.12 \mathrm{ppm}$ in the ${ }^{29} \mathrm{Si}$ NMR spectrum (Fig. S2) indicated that the silicon atoms were all magnetically equivalent, and the T-type silanol silicon peak confirmed that each silicon atom was covalently bonded with three oxygen atoms and one carbon atom. The asymmetric stretching vibration of $\mathrm{Si}-\mathrm{O}-\mathrm{Si}$ at $1082 \mathrm{~cm}^{-1}$ and $1000 \mathrm{~cm}^{-1}$ in FTIR confirmed the cage-like structure as well (Fig. S1). As shown in Scheme 1b, ProDOT-ene was synthesized via p-TSA catalyzed transetherification from3,4-dimethioxythiophene and 2-allyl-1,3-propanediol. The ProDOT-ene was purified as a light yellow viscous liquid and the chemical structure was confirmed by NMR and FTIR. In Figure 1b, all the chemical shifts corresponding to each proton on ProDOT-ene are clearly assigned. The two peaks located at $5.2 \mathrm{ppm}$ and $5.8 \mathrm{ppm}$ in ${ }^{1} \mathrm{H}$ NMR correspond to the protons on the ene moiety. The electroactive cross linker, POSS-ProDOT, was prepared from POSS-SH and ProDOT-ene via thiol-ene "click" chemistry with $0.1 \mathrm{w} \%$ DMPA as radical initiator. ${ }^{32}$ As was confirmed by both ${ }^{1} \mathrm{H}$ NMR (Fig. 1c) and FTIR (Fig. S1), the disappearance of the three peaks located at $1.38 \mathrm{ppm}, 5.2$ $\mathrm{ppm}$ and $5.8 \mathrm{ppm}$ in ${ }^{1} \mathrm{H}$ NMR and changes of the characteristic peaks associated with the alkene group on the ProDOT-ene $\left(=\mathrm{C}-\mathrm{H}\right.$ bend at 916 and $994 \mathrm{~cm}^{-1}$ and $\mathrm{C}=\mathrm{C}$ stretch around $1639 \mathrm{~cm}^{-1}$ ) and the -SH group on the POSS-SH (-SH bend at $\left.2550 \mathrm{~cm}^{-1}\right)$ indicated the success of the thiol-ene reaction.

\subsection{Electrochemical studies of POSS-ProDOT, EDOT and EDOT/POSS-ProDOT mixed solutions}

The electrochemical behavior of POSS-ProDOT crosslinker was investigated via Cyclic Voltammetry $(\mathrm{CV})$ by potentiodynamically scanning the solution between $-0.6 \mathrm{~V}$ and $+1.5 \mathrm{~V}$ (vs. $\mathrm{Ag} / \mathrm{AgNO}_{3}$ ). Cyclic Voltammograms from a typical experiment are shown in Figure 2. An irreversible oxidation corresponding to thiophene ring from the POSS-ProDOT that occurred at $+1.20 \mathrm{~V}$ was seen in the first anodic scan (red curve). The fact that the oxidation 
potential of the cross linker was similar to that of EDOT $^{33}$ indicated that it would be relatively easy to get POSS-ProDOT incorporated into PEDOT films. In the subsequent scans, unlike the electrochemical polymerization of ProDOT derivatives, no obvious peaks associated with the formation of PProDOT polymers were found at lower potentials during both anodic and cathodic scans. ${ }^{15}$ This low polymerizability of POSS-ProDOT is presumably due to the large steric hindrance of the bulky POSS core. It has been reported by several groups that monomers with bulky side groups cannot form their corresponding polymers via direct electrochemical polymerization. ${ }^{15,34}$ The electrochemical behaviors of pure EDOT and EDOT/POSS-ProDOT mixed solutions were also studied via cyclic voltammetry and no obvious differences were observed among EDOT and various EDOT/ POSS-ProDOT solutions. Hundreds of nanometers of blue shifts in absorption maxima were observed with $\mathrm{EPh}$ as crosslinker, leading to dramatic changes in color. ${ }^{12}$ The blue shift of absorption was resulted from the decreased conjugated chain length which could potentially lead to an eventual loss in charge transport efficiency, usually larger blue shift could result in more loss of conductivity. However, in this system, much smaller shifts in absorption were observed. These results indicate that the POSS-ProDOT crosslinker does not significantly disrupt the overall conjugation of the PEDOT backbone, making it possible to maintain the desirable electronic properties. ${ }^{35,36}$

\subsection{Optical Properties}

PEDOT/POSS-ProDOT copolymer films were obtained by applying a potentiodynamic scan with potential cycling between $-0.6 \mathrm{~V}$ and $+1.2 \mathrm{~V}$ ( vs. $\mathrm{Ag} / \mathrm{AgNO}_{3}$ ) for 8 cycles in a series of mixed monomer solutions. The optical properties of the films prepared in mixed monomer solutions didn't change significantly with increasing POSS-ProDOT feed ratio. The electrochemically deposited PEDOT had a blue color due to its partially oxidized state, and the copolymers showed similar colors as the PEDOT thin films. The UV-Vis spectra of the polymer films are shown in Figure S3. All the films were reduced at $-1.0 \mathrm{~V}$ for 1 minute before measurement and they showed an increasing trend of absorption from 400 to $800 \mathrm{~nm}$, with a local absorption maximum near $600 \mathrm{~nm}$. It was found that the copolymerization shifted the absorption maximum to shorter wavelength. As shown in Table 1, for pure PEDOT thin films, the absorption maximum was $576 \mathrm{~nm}$, while with more and more crosslinker added to the solution, the absorption maximum shifted from $576 \mathrm{~nm}$ to $543 \mathrm{~nm}$. The modest $33 \mathrm{~nm}$ difference in the absorption maxima also explains the similar blue colors of the pure PEDOT and PEDOT-co-POSS-ProDOT copolymer films. It was previously reported that the absorption maxima of PEDOT films could be significantly tuned by adding a conjugated crosslinker, EPh.

\subsection{Electrochemical Properties}

PEDOT and PEDOT-co-POSS-ProDOT copolymers were electrochemically polymerized onto stainless steel electrodes under constant voltage $(+1.1 \mathrm{~V}$, a total deposition charge of 27 $\mathrm{mC}$ ) with tetrabutylammonium perchlorate (TBAP, $0.1 \mathrm{M}$ ) as electrolyte in a standard threeelectrode cell. We performed both cyclic voltammetry (CV) to determine the charge storage Capacity (CSC) and electrical impedance spectroscopy (EIS) to determine electrode impedance. Cyclic voltammetry of stainless steel electrodes, PEDOT and PEDOT-co-POSSProDOT copolymers coated electrodes are shown in Figure 3a. Both PEDOT and its 
copolymers showed oxidation and reduction reactions as indicated by the anodic and cathodic current peaks on the $\mathrm{CV}$ curve. The peak potential indicates the voltage at which the reaction takes place and the enclosed areas of the curve are proportional to the charge storage capacity. Clearly the PEDOT and the copolymer films have significantly higher charge storage capacities than the uncoated stainless steel electrode. The CV curves of PEDOT and copolymers were of similar shape, while the oxidation peaks shifted to the positive direction with increasing amounts of crosslinker. These shifted oxidation peaks indicated that the crosslinker decreased the conjugation length of PEDOT. ${ }^{12,}, 35,37$

The electrochemical properties of coated electrodes were further investigated using electrical impedance spectroscopy (EIS) in 0.1 M PBS buffer solution. After PEDOT deposition, the amplitude of the impedance of the coated electrodes decreased by 2-3 orders of magnitude (from $\sim 10000 \Omega$ to $\sim 100 \Omega$ ) at low frequencies as seen in Figure 3b. This dramatic drop in impedance has been associated with the increase in effectivee surface area and ability of the PEDOT to facilitate both electronic and ionic charge transport. ${ }^{38,} 39$ It was also found that at low POSS-ProDOT concentration $(<3.1 \mathrm{wt} \%)$, the impedance of PEDOT copolymer coated electrodes was similar to the PEDOT-coated electrodes, which were all around $100 \Omega$. The impedance was slightly increased from $100 \Omega$ to $200 \Omega$ for PEDOT films with higher POSSProDOT concentration $(8.0 \mathrm{wt} \%)$. The increased impedance at higher crosslinker concentration likely comes from three major factors. First of all, POSS nanomaterials are insulating and the excess POSS raises the internal impedance of the PEDOT-8.0 wt\% POSSProDOT. In addition, the decreased conjugation chain length may be another reason for the increasing impedance. When $1.7 \%$ POSS-ProDOT was added, the absorption maximum blue-shifted $13 \mathrm{~nm}$, and a small change in impedance was observed. However, as the feeding ration up to $3.1 \%$, the absorption maximum blue-shifted $27 \mathrm{~nm}$, indicating the conjugation length shortened more significantly, therefore, large variation on impedance can be found. Last but not least, as discussed in more detail in the following section, the crosslinker changed the morphology of the polymer film during the electrochemical deposition as shown in Figure 4. As increasing the feed ratio of POSS-ProDOT, the morphology of the film became denser, resulting in a higher impedance. It has been reported that films with an open structure usually have lower impedances in comparison to the films with a closed, dense structure. ${ }^{40}$ The $8.0 \mathrm{wt} \%$ POSS-ProDOT feed ratio had the highest crosslinker concentration in the film and also had the highest impedance. The phase plot of the impedance spectroscopy revealed phase angles of $65-80^{\circ}$ for the bare stainless steel electrodes at frequencies of less than $1000 \mathrm{~Hz}$ which indicates that the electrode is primarily functioning as a capacitor (Figure 3c). PEDOT coatings dramatically dropped the phase angles to a value below $20^{\circ}$ making the electrodes more resistive as opposed to capacitive at frequencies above $5 \mathrm{~Hz}$. There was a similar trend of the phase angle change for PEDOT and copolymers with low crosslinker concentration $(<3.1 \mathrm{wt} \%)$ while it was different for the copolymer film with $8.0 \mathrm{wt} \%$ crosslinker indicating that there was also a structure change in the film.

\subsection{Electrochemical stability}

To determine their long-term electrochemical stability, the conjugated polymer coatings were studied by repeated CV tests in a PBS buffer solution at a scan rate of $100 \mathrm{mV} / \mathrm{s}$ for 
1000 consecutive cycles. After the CV cycling, the polymer films were subjected to EIS testing. Figure 5 clearly shows that the copolymerization significantly affected the electrochemical stability of PEDOT films. With $1.7 \mathrm{wt} \%$ POSS-ProDOT in the feed, the copolymer film showed similar impedance spectra $(<3000 \Omega)$ as the PEDOT film at the same deposition charge density. By increasing the POSS-ProDOT feed ratio to $3.1 \mathrm{wt} \%$, a significant decrease in impedance magnitude $(\sim 400 \Omega)$ was observed in comparison to the pure PEDOT film. When the POSS-ProDOT feed ratio was further increased to $8.0 \mathrm{wt} \%$, the impedance benefit from the conjugated polymer coatings was again diminished. At $8.0 \mathrm{wt} \%$ POSS-ProDOT feed ratio, the impedance magnitude (> $10000 \Omega$ ) of the coated electrode after the stability test was the same as the uncoated stainless steel electrode. The results are presumably due to the continuous swelling and shrinkage during the long-term charge/ discharge process. The POSS-ProDOT crosslinks the PEDOT film, restricting the swelling and shrinkage of the polymer films during the oxidation and reduction process. For $1.7 \mathrm{wt} \%$ POSS-ProDOT feed ratio, only a few cross-linkers were incorporated into the polymer and thus the copolymer showed similar electrochemical stability as PEDOT. By increasing the feed ratio to $3.1 \mathrm{wt} \%$, an intermediate amount of POSS-ProDOT was incorporated into the polymer and the strengthening effect helps to increase the stability of PEDOT coatings. At higher feed ratios $(8.0 \mathrm{wt} \%$ ) brittleness was observed, probably due to the high extent of intermolecular bonding from the excessive crosslinking within the polymer matrix. During the $\mathrm{CV}$ stimulations, ion exchange between the electrode and electrolyte lead to a certain amount of volumetric strain to the polymer coatings. This will lead to cracking and delamination if the associated strains are larger than the polymer coating's inherent strain to failure. The stability change of copolymer films was also confirmed by evaluating the morphology of the coatings after CV cycling by SEM. A comparison of the electrochemical impedance of stainless steel, PEDOT and copolymers at $1 \mathrm{~Hz}$ before and after stability tests is shown in Figure 5b. The SEM images of the PEDOT and copolymer with various POSSProDOT contents are shown in Figure 6. After the stability test, PEDOT film showed cracking and delamination which is consistent with literature. ${ }^{20,21}$ By adding $1.7 \mathrm{wt} \%$ POSS-ProDOT, cracking was still observed but their sizes were much smaller. Films with $3.1 \mathrm{wt} \%$ POSS-ProDOT showed the most intact morphology with no obvious cracking or delamination. Polymer coatings with $8.0 \mathrm{wt} \%$ showed extensive cracking and delamination, similar to the pure PEDOT films. The trend of the effect of cross linker on the morphologies and electrochemical stability of PEDOT films correlated well with the EIS spectra.

\section{Conclusions}

In summary, we have synthesized an octa-ProDOT functionalized polyhedral oligomeric silsesquioxane (POSS-ProDOT) crosslinker via hydrosilylation and thiol-ene "click" chemistry. Its molecular structure was confirmed by both nuclear magnetic resonance (NMR) $\left({ }^{1} \mathrm{H},{ }^{13} \mathrm{C},{ }^{29} \mathrm{Si}\right.$ ) and Fourier transform infrared (FT-IR) spectroscopies. The crosslinker was electrochemically copolymerized with EDOT in mixed monomer solutions to create cross-linked PEDOT: POSS-ProDOT copolymer films on metal electrodes. The optical, electrical and morphological properties of the copolymer films can be tuned by adjusting the feeding ratio of the cross linker. With more POSS-ProDOT incorporated into the films, the optical absorption shifted towards shorter wavelength direction, the impedance 
slightly increased and the morphology gradually changed from an open structure to a more closed structure. Significantly enhanced electrochemical stability was observed with the addition of POSS-ProDOT, with the optimum performance at $3.1 \mathrm{wt} \%$ POSS-ProDOT. Further additions of POSS-ProDOT led to the development of brittleness, as seen by cracking of the film. It is expected that these highly stable PEDOT-co-POSS-ProDOT materials will be excellent candidates for use in bioelectronic devices such as neural electrodes.

\section{Supplementary Material}

Refer to Web version on PubMed Central for supplementary material.

\section{Acknowledgments}

We thank the National Science Foundation (DMR-1103027, DMR-1505144), the National Institutes of Health through EUREKA grant 1RO1EB010892, the Defense Advanced Research Projects Agency (DARPA) grant N66001-11-C-4190, and the University of Delaware for funding. We thank Nandita Bhagwat for providing the ProDOT-ene monomer.

\section{References}

1. Cosnier S. Anal Bioanal Chem. 2003; 377:507. [PubMed: 12920501]

2. Long YZ, Li MM, Gu C, Wan M, Duvail J, Liu Z, Fan Z. Prog Polym Sci. 2011

3. Luo X, Weaver CL, Zhou DD, Greenberg R, Cui XT. Biomaterials. 2011; 32:5551. [PubMed: 21601278]

4. Wilks SJ, Woolley AJ, Ouyang L, Martin DC, Otto KJ. Conf Proc IEEE Eng Med Biol Soc. 2011:5412. [PubMed: 22255561]

5. Smela E. Adv Mater. 2003; 15:481.

6. Balint R, Cassidy NJ, Cartmell SH. Acta Biomater. 2014; 10:2341. [PubMed: 24556448]

7. Zhu B, Luo SC, Zhao H, Lin HA, Sekine J, Nakao A, Chen C, Yamashita Y, Yu Hh. Nat Commun. 2014; 5:4523. [PubMed: 25060339]

8. Zhao H, Zhu B, Sekine J, Luo SC, Yu Hh. ACS Appl Mater Interfaces. 2012; 4:680. [PubMed: 22211371]

9. Martin DC. MRS Commun. 2015; 5:131.

10. Liu J, Wei B, Sloppy JD, Ouyang L, Ni C, Martin DC. ACS Macro Lett. 2015; 4:897.

11. Green RA, Lovell NH, Wallace GG, Poole-Warren LA. Biomaterials. 2008; 29:3393.

12. Ouyang L, Kuo CC, Farrell B, Pathak S, Wei B, Qu J, Martin DC. J Mater Chem B. 2015; 3:5010.

13. Green RA, Abidian MR. Adv Funct Mater. 2015; 27:7620.

14. Wei B, Liu J, Ouyang L, Kuo CC, Martin DC. ACS Appl Mater Interfaces. 2015; 7:15388. [PubMed: 26052833]

15. Wei B, Ouyang L, Liu J, Martin DC. J Mater Chem B. 2015; 3:5028.

16. Martin DC, Wu J, Shaw CM, King Z, Spanninga SA, Richardson-Burns S, Hendricks J, Yang J. Polym Rev. 2010; 50:340.

17. Abidian MR, Ludwig KA, Marzullo TC, Martin DC, Kipke DR. Adv Mater. 2009; 21:3764. [PubMed: 26345408]

18. Abidian MR, Martin DC. Adv Funct Mater. 2009; 19:573.

19. Frost CM, Wei B, Baghmanli Z, Cederna PS, Urbanchek MG. Plast Reconstr Surg. 2012; 129:933. [PubMed: 22456363]

20. Kung TA, Langhals NB, Martin DC, Cederna PS, Urbanchek MG. Plast Reconstr Surg. 2014; 133:1380. [PubMed: 24867721] 
21. Green RA, Hassarati RT, Bouchinet L, Lee CS, Cheong GL, Yu JF, Dodds CW, Suaning GJ, PooleWarren LA, Lovell NH. Biomaterials. 2012; 33:5875. [PubMed: 22656446]

22. Ghosh S, Inganas O. J Electrochem Soc. 2000; 147:1872.

23. Ghosh S, Inganäs O. Adv Mater Commun. 1999; 11:1214.

24. Khodagholy D, Doublet T, Gurfinkel M, Quilichini P, Ismailova E, Leleux P, Herve T, Sanaur S, Bernard C, Malliaras GG. Adv Mater. 2011; 23:268. [PubMed: 21154741]

25. Wei B, Liu J, Ouyang L, Kuo CC, Martin DC. ACS Appl Mater Interfaces. 2015; 7:15388. [PubMed: 26052833]

26. Carli S, Casarin L, Bergamini G, Caramori S, Bignozzi CA. J Phys Chem C. 2014; 118:16782.

27. Jerman I, Vuk AS, Kozelj M, Orel B, Kovac J. Langmuir. 2008; 24:5029. [PubMed: 18351793]

28. Miyake J, Sawamura T, Kokado K, Chujo Y. Macromol Rapid Commun. 2009; 30:1559. [PubMed: 21638421]

29. Li Y, Zhang Q, Zhao X, Yu P, Wu L, Chen D. J Mater Chem. 2012; 22:1884.

30. Wang W, Chen M, Chen X, Wang J. Chem Eng J. 2014; 242:62.

31. Luo A, Jiang X, Lin H, Yin J. J Mater Chem. 2011; 21:12753.

32. Feldman KE, Martin DC. Biosensors. 2012; 2:305. [PubMed: 25585932]

33. Groenendaal L, Zotti G, Aubert PH, Waybright SM, Reynolds JR. Adv Mater. 2003; 15:855.

34. Bu HB, Gotz G, Reinold E, Vogt A, Schmid S, Blanco R, Segura JL, Bauerle P. Chem Commun. 2008:1320.

35. Qin L, Xu J, Lu B, Lu Y, Duan X, Nie G. J Mater Chem. 2012; 22:18345.

36. Li M, Sheynin Y, Patra A, Bendikov M. Chem Mater. 2009; 21:2482.

37. Lin C, Endo T, Takase M, Iyoda M, Nishinaga T. J Am Chem Soc. 2011; 133:11339. [PubMed: 21678981]

38. Cui X, Martin DC. Sens Actuators B: Chem. 2003; 89:92.

39. Martin DC, Malliaras GG. ChemElectroChem. 2016; 3:686.

40. Cui X, Martin DC. Sens Actuators A: Phys. 2003; 103:384. 

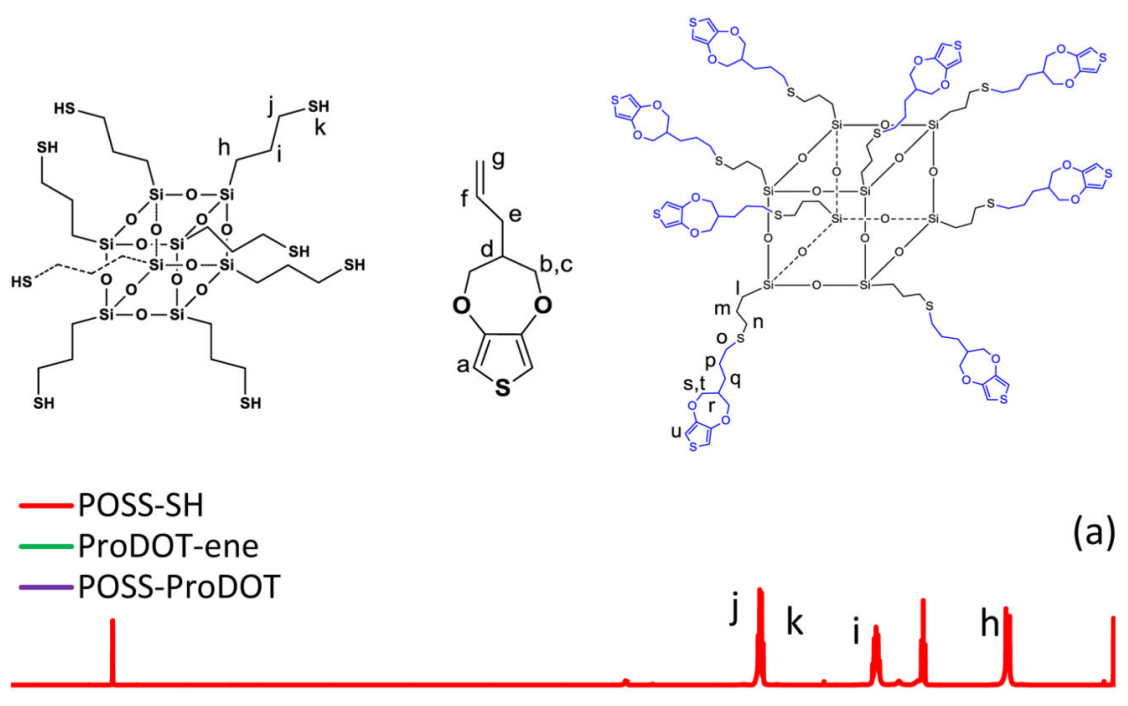

(a)

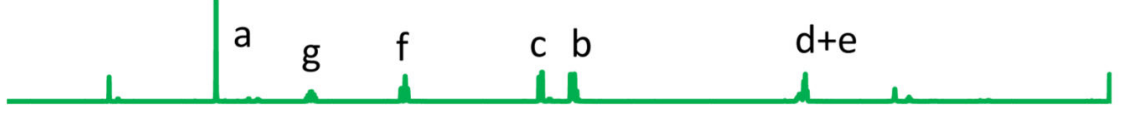

(c)

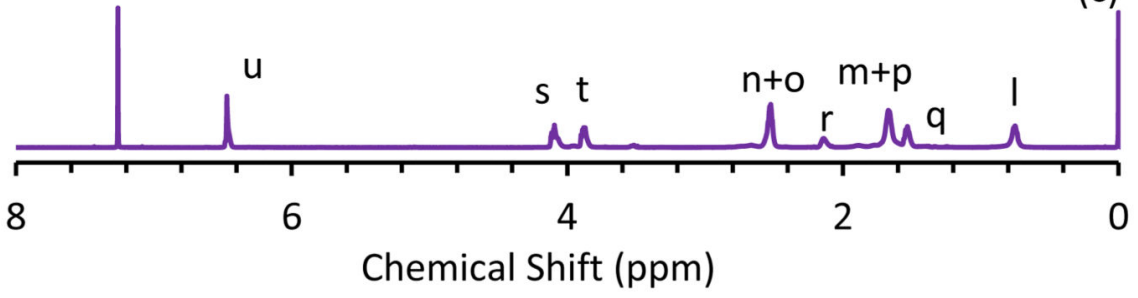

Figure 1.

Chemical structure and ${ }^{1} \mathrm{H}$ NMR spectra in CDCl3. (a) POSS-SH, (b) ProDOT-ene and (c) POSS-ProDOT 


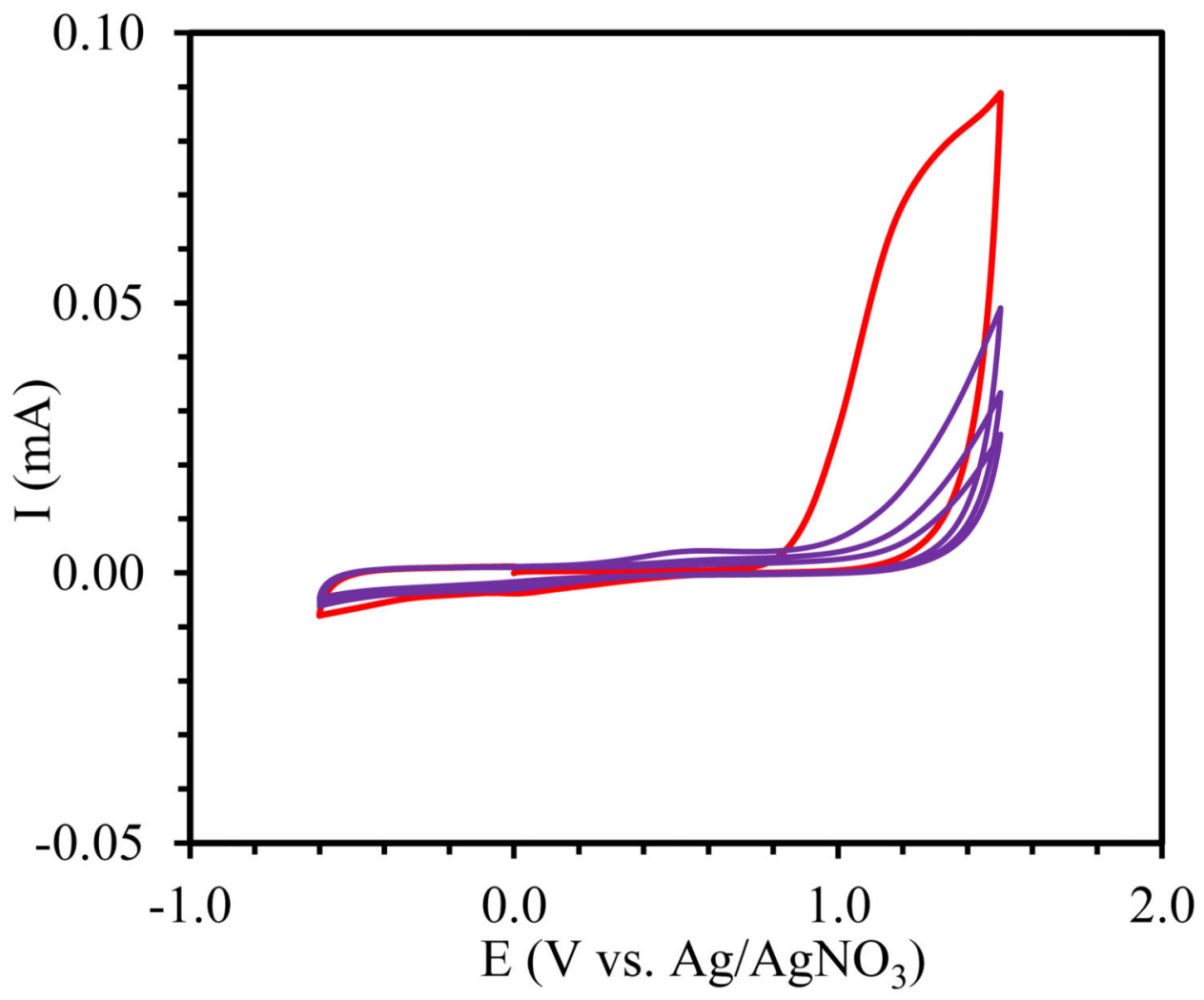

Figure 2.

Electrochemical behavior of POSS-ProDOT. The POSS-ProDOT crosslinker solution $(5 \mathrm{mM})$ was potential dynamically scanned from $-0.6 \mathrm{~V}$ to $+1.4 \mathrm{~V}$ in ACN/TBAP $(0.1 \mathrm{M})$ for 4 cycles. An oxidation onset at $+1.0 \mathrm{~V}$ was observed at the 1 st scan (red), no oxidation peaks corresponding to the formation of polymer was observed in the subsequent scans, 2nd to 4 th (purple). 

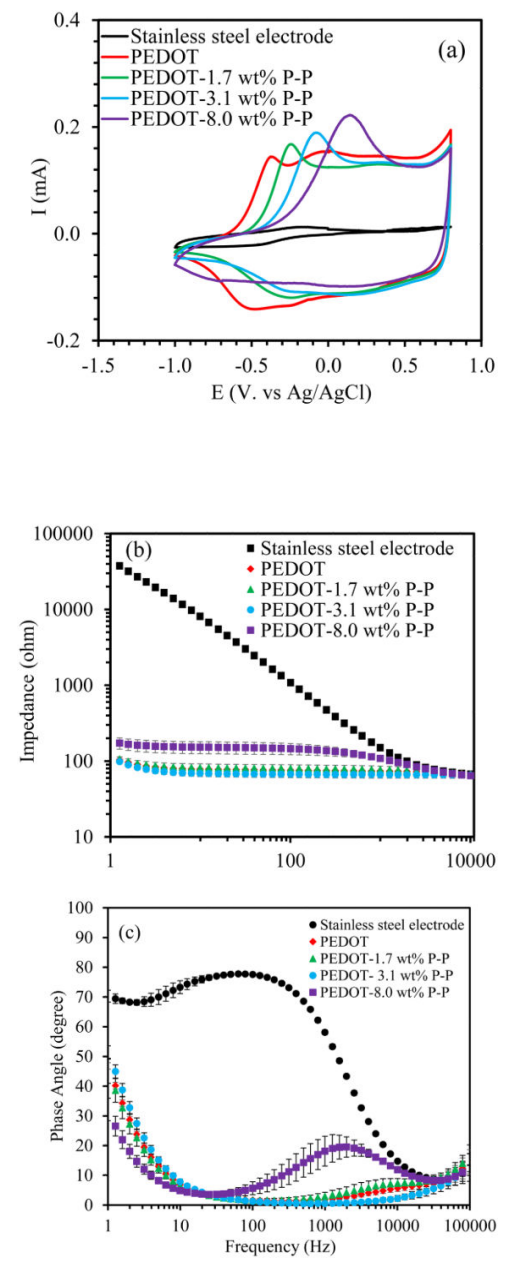

Figure 3.

Cyclic voltammetry (CV) and Electrochemical impedance spectroscopy (EIS). (a) The CV curves of PEDOT and copolymer films were of similar shape while their charge storage capacities are significantly larger than that of stainless steel electrode. The oxidation peaks of these polymer films shifted to the positive direction with increasing amounts of crosslinker. These shifted oxidation peaks indicated that the crosslinker changed the conjugation length of PEDOT. (b) EIS of stainless steel, PEDOT and copolymer films deposited on stainless steel. The minor increase of impedance at high crosslinker concentration $(8.0 \mathrm{wt} \%)$ indicated that crosslinker only has a slight effect on the impedance of the films. (Each point of EIS value was obtained by 3 measurements, and error bars show standard deviations). (c) Phase Angle of the EIS of stainless steel, PEDOT and copolymer films deposited on stainless steel. Stainless steel electrode is primarily functioning as a capacitor, and these polymer films are more like resistors. 

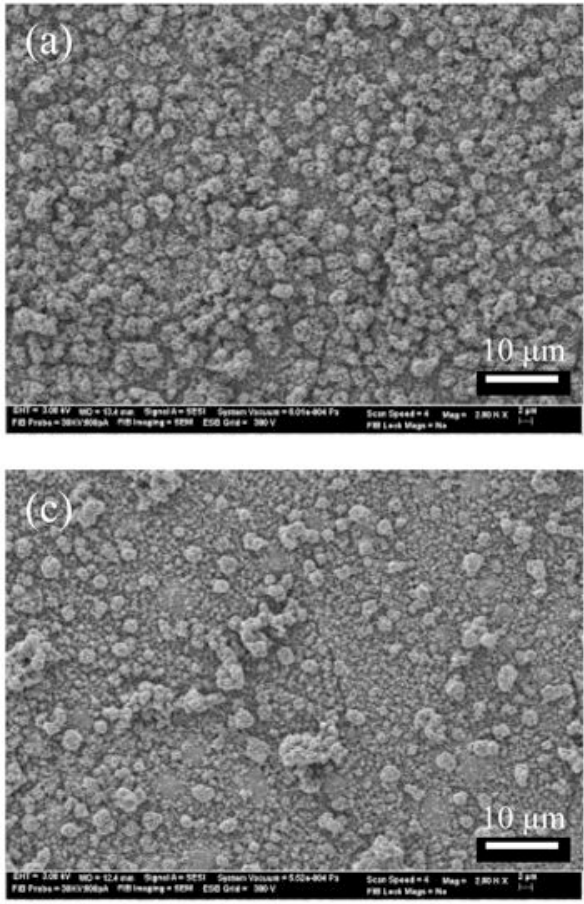
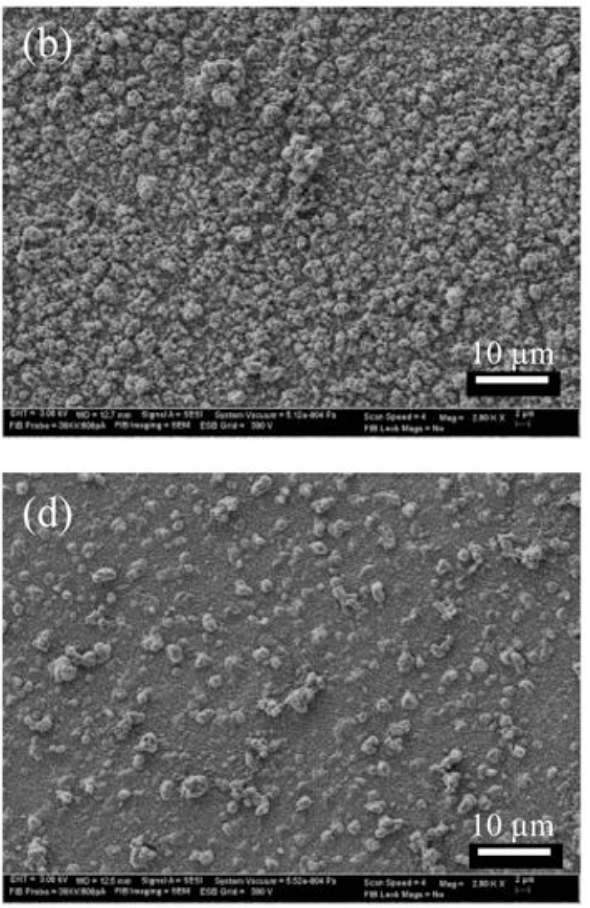

Figure 4.

SEM images of PEDOT and copolymer films. (a) PEDOT; (b) PEDOT-1.7 wt\% P-P; (c) PEDOT-3.1 wt\% P-P; (d) PEDOT-8.0 wt\% P-P. (Scale bar represents $10 \mu \mathrm{m}$ ) 

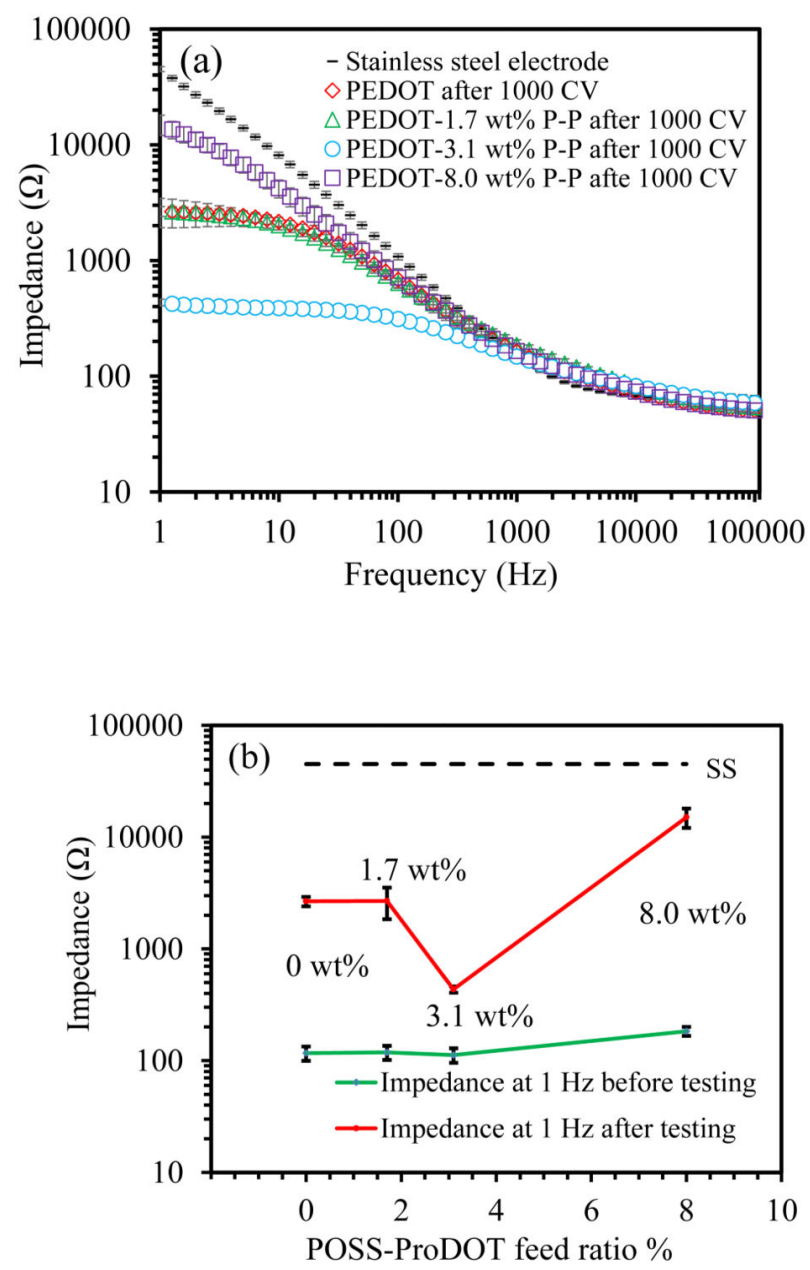

Figure 5.

Electrochemical impedance spectroscopy of PEDOT and copolymer films after stability test. (a) EIS after stability test showed that PEDOT with $3.1 \mathrm{wt} \%$ crosslinker performed the best. (b) Comparison of impedance at $1 \mathrm{~Hz}$ of PEDOT and copolymer films before and after stability test. (Each point of EIS value was obtained by 3 measurements, and error bars show standard deviations) 

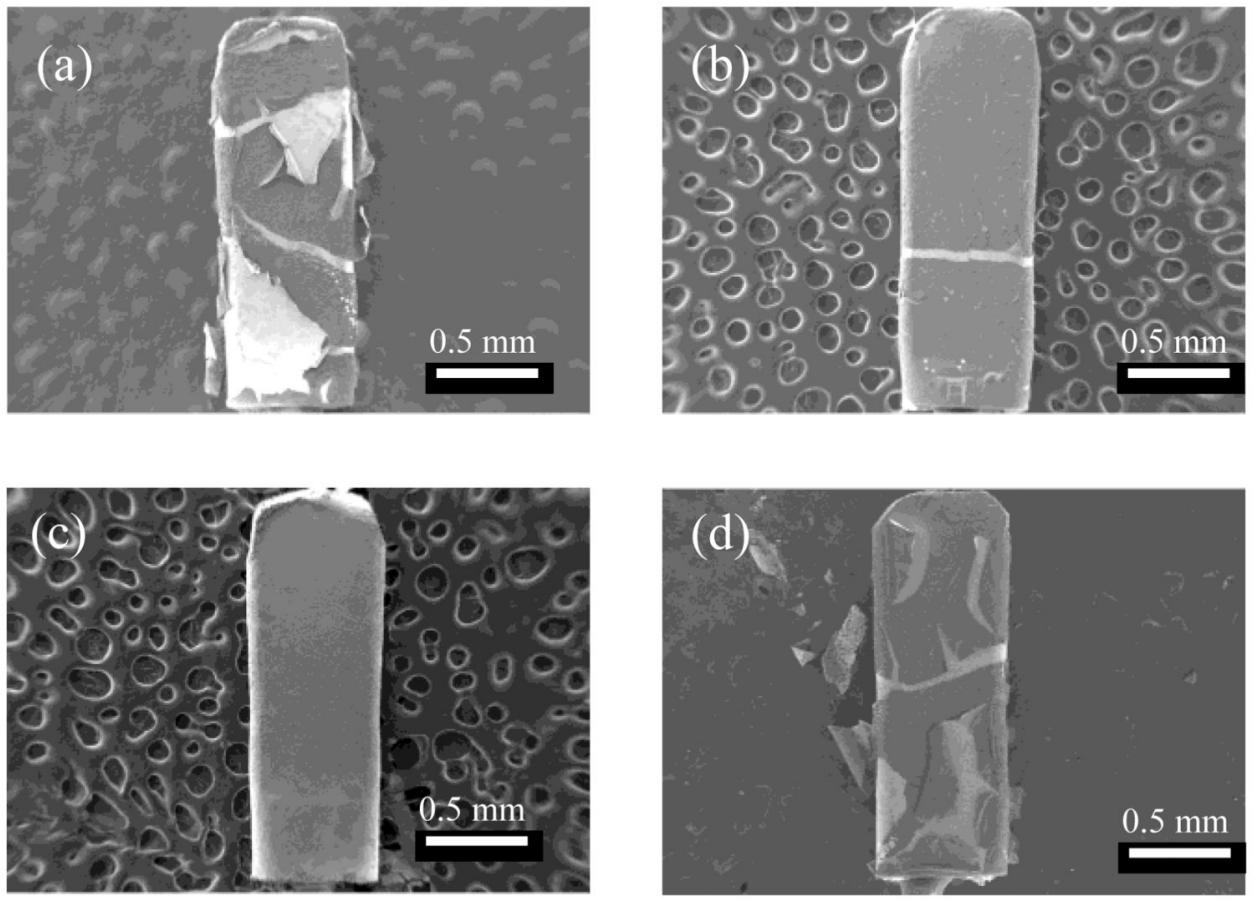

Figure 6.

Morphologies of PEDOT and copolymer films after stability test: (a) Both cracking and delamination were observed on PEDOT film; (b) Only cracking was found on PEDOT film with 1.7 wt \% crosslinker; (c) No cracking nor delamination was found on PEDOT with 3.1 wt $\%$ crosslinker; (d) Both cracking and delamination were observed on PEDOT film with $8.0 \mathrm{wt} \%$ crosslinker. (Scale bar represents $0.5 \mathrm{~mm}$ ) 
(a)

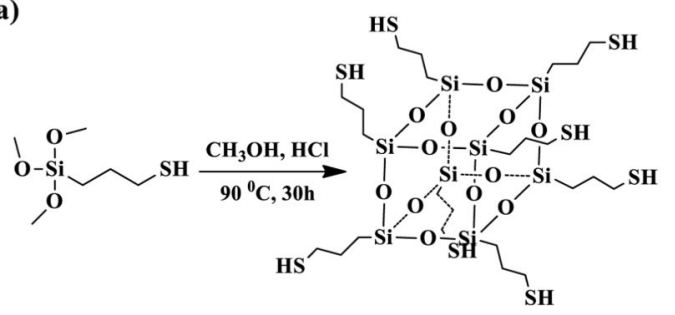

(b)

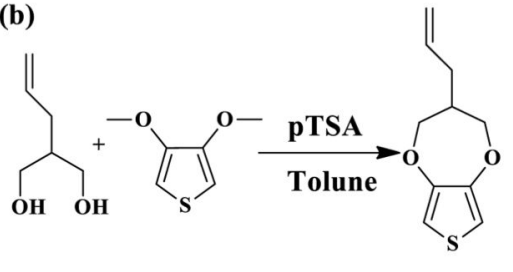

(c)

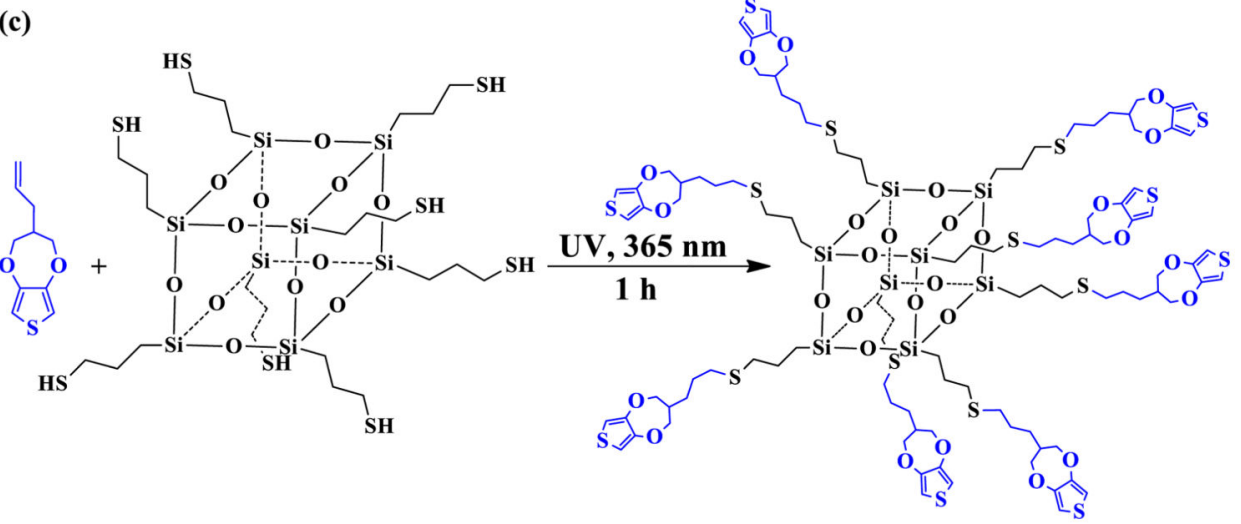

Scheme 1.

Syntheses of POSS-ProDOT crosslinker. (a) Synthesis of POSS-SH via hydrosilylation. (b) Synthesis of ProDOT-ene. (c) Synthesis of POSS-ProDOT via thiol-ene "click" chemistry 


\section{Table 1}

UV-vis absorption maxima. The absorption maxima difference of reduced PEDOT and copolymer films indicated that crosslinker does not significantly disrupt the overall conjugation of the PEDOT backbone.

\begin{tabular}{lc}
\hline Polymer & Wavelength [nm] \\
\hline PEDOT & 576 \\
\hline PEDOT-1.7 wt\% P-P & 563 \\
\hline PEDOT-3.1 wt\% P-P & 549 \\
\hline PEDOT-8.0 wt\% P-P & 543 \\
\hline
\end{tabular}

